\title{
First Universal Newborn Screening Program for Severe Combined Immunodeficiency in Europe. Two-Years' Experience in Catalonia (Spain)
}

\section{OPEN ACCESS}

Edited by:

Antonio Condino-Neto,

University of São Paulo, Brazil

Reviewed by:

Raz Somech,

Sheba Medical Center, Israel

Lisa Renee Forbes,

Baylor College of Medicine,

United States

Mirjam van der Burg,

Leiden University Medical

Center, Netherlands

*Correspondence:

Pere Soler-Palacín

psoler@vhebron.net

tThese authors have contributed equally to this work

Specialty section:

This article was submitted to

Primary Immunodeficiencies,

a section of the journal

Frontiers in Immunology

Received: 27 May 2019 Accepted: 25 September 2019

Published: 22 October 2019

Citation:

Argudo-Ramírez A, Martín-Nalda A, Marín-Soria JL, López-Galera RM,

Pajares-García S, González de Aledo-Castillo JM, Martínez-Gallo M,

García-Prat M, Colobran R,

Riviere JG, Quintero Y, Collado T,

García-Villoria J, Ribes A and Soler-Palacín P (2019) First Universal

Newborn Screening Program for Severe Combined Immunodeficiency in Europe. Two-Years' Experience in

Catalonia (Spain).

Front. Immunol. 10:2406.

doi: 10.3389/fimmu.2019.02406
Ana Argudo-Ramírez ${ }^{1+}$, Andrea Martín-Nalda ${ }^{2+}$, Jose L. Marín-Soria ${ }^{1}$, Rosa M. López-Galera ${ }^{1}$, Sonia Pajares-García ${ }^{1}$, Jose M. González de Aledo-Castillo ${ }^{1}$, Mónica Martínez-Gallo ${ }^{3}$, Marina García-Prat ${ }^{2}$, Roger Colobran ${ }^{3,4}$, Jacques G. Riviere ${ }^{2}$, Yania Quintero ${ }^{1}$, Tatiana Collado ${ }^{1}$, Judit García-Villoria ${ }^{1}$, Antonia Ribes ${ }^{1}$ and Pere Soler-Palacín ${ }^{2 *}$

${ }^{1}$ Newborn Screening Laboratory, Inborn Errors of Metabolism Division, Biochemistry and Molecular Genetics Department, Hospital Clínic, Barcelona, Spain, ${ }^{2}$ Pediatric Infectious Diseases and Immunodeficiencies Unit, Jeffrey Modell Diagnostic and Research Center for Primary Immunodeficiencies, Hospital Universitari Vall d'Hebron, Universitat Autònoma de Barcelona, Barcelona, Spain, ${ }^{3}$ Immunology Division, Jeffrey Modell Diagnostic and Research Center for Primary Immunodeficiencies, Hospital Universitari Vall d'Hebron, Universitat Autònoma de Barcelona, Barcelona, Spain, ${ }^{4}$ Department of Clinical and Molecular Genetics, Jeffrey Modell Diagnostic and Research Center for Primary Immunodeficiencies, Hospital Universitari Vall d'Hebron, Universitat Autònoma de Barcelona, Barcelona, Spain

Severe combined immunodeficiency (SCID), the most severe form of T-cell immunodeficiency, can be screened at birth by quantifying T-cell receptor excision circles (TRECs) in dried blood spot (DBS) samples. Early detection of this condition speeds up the establishment of appropriate treatment and increases the patient's life expectancy. Newborn screening for SCID started in January 2017 in Catalonia, the first Spanish and European region to universally include this testing. The results obtained in the first 2 years of experience are evaluated here. All babies born between January 2017 and December 2018 were screened. TREC quantification in DBS (1.5 mm diameter) was performed with the Enlite Neonatal TREC kit from PerkinElmer (Turku, Finland). In 2018, the retest cutoff in the detection algorithm was updated based on the experience gained in the first year, and changed from 34 to 24 copies $/ \mu \mathrm{L}$. This decreased the retest rate from 3.34 to $1.4 \%$ (global retest rate, $2.4 \%$ ), with a requested second sample rate of $0.23 \%$ and a positive detection rate of $0.02 \%$. Lymphocyte phenotype (T, B, NK populations), expression of CD45RA/RO isoforms, percentage and intensity of TCR $\alpha \beta$ and TCR $\gamma \delta$, presence of HLA-DR+ T lymphocytes, and in vitro lymphocyte proliferation were studied in all patients by flow cytometry. Of 130,903 newborns screened, 30 tested positive, 15 of which were male. During the study period, one patient was diagnosed with SCID: incidence, 1 in 130,903 births in Catalonia. Thirteen patients had clinically significant T-cell lymphopenia (non-SCID) with an incidence of 1 in 10,069 newborns (43\% of positive detections). Nine patients were considered false-positive cases because of an initially normal lymphocyte count with normalization of TRECs between 3 and 6 months of life, four infants had transient lymphopenia due to an initially 
low lymphocyte count with recovery in the following months, and three patients are still under study. The results obtained provide further evidence of the benefits of including this disease in newborn screening programs. Longer follow-up is needed to define the exact incidence of SCID in Catalonia.

Keywords: newborn screening, severe combined immunodeficiency, T-cell receptor excision circles, T-cell receptor, T-lymphocytes, stem cell transplantation

\section{INTRODUCTION}

Newborn screening (NBS), initially implemented in the United States in 1961 (1), has been available in most developed countries for decades, originally for the detection and early treatment of phenylketonuria and now for other endocrine and metabolic diseases, mainly using tandem mass spectrometry. DNA-based testing for other diseases has been only recently included in the NBS programs of some countries. Since its initial implementation in Wisconsin in 2008, NBS for severe combined immunodeficiency (SCID) using a T-cell receptor excision circle (TREC) assay has been established worldwide, including in most of the United States (2), Taiwan (3), Israel (4), New Zealand, some Canadian regions (2), Norway (5), and Catalonia (Spain). Previously published results showed an incidence of SCID of around 1 in 56,000 newborns $(5,6)$ and a high survival rate $(92 \%)$ after appropriate treatment (2).

SCID, the most severe form of T-cell primary immunodeficiency (PID), includes a group of inherited defects characterized by severe T-cell lymphopenia (TCL). Patients with SCID require prompt clinical intervention to prevent severe life-threatening infections, and several studies have reported significantly improved survival in babies diagnosed at birth $(7,8)$. Curative treatment is based on hematopoietic stem cell transplantation (HSCT) or gene therapy when available (8). SCID can be screened at birth in a cost-effective way on a large scale through quantification of T-cell receptor excision circles (TRECs) in Guthrie card dried blood spot (DBS) samples $(9,10)$.

SCID newborn screening can also identify other clinically relevant forms of TCL, which have an overall incidence of 1 in 7,300 newborns (2). These include specific syndromes such as 22q11 deletion (DiGeorge) syndrome, Down syndrome, and CHARGE syndrome among others $(11,12)$.

TRECs are circular DNA molecules formed by T-cell receptor gene rearrangement during the normal process of T-cell differentiation in the thymus. TRECs do not undergo further replication in dividing cells. Hence, they are stable circular DNA fragments, which are a useful marker of recently formed T-cells, a cell population that is extremely low in newborns with SCID. TREC copy number can be determined using a quantitative PCR-based method with time-resolved fluorescence resonance energy transfer (TR-FRET)-based detection in eluted DNA from routinely collected DBS (9). Kappa-deleting recombination excision circles (KRECs), the circular by-product of $\mathrm{B}$ cell immunoglobulin kappa gene rearrangement, have been proposed for a combined TREC-KREC screening approach (13). This could also enable the early detection of patients with severe forms of B cell deficiency, such as X-linked agammaglobulinemia (XLA) (14).

The implementation of screening through TREC assays provides the earliest possible identification and allows for prompt, successful transplantation before infants experience severe infection, organ damage, and, ultimately, death (15). The cost-effectiveness of including SCID in NBS programs has been demonstrated in the United States $(16,17)$ and Europe $(6,10,18,19)$.

In Catalonia, the NBS program started with phenylketonuria detection in 1969 and congenital hypothyroidism in 1982. Today, around 67,000 newborns per year are screened for 24 diseases (phenylketonuria, congenital hypothyroidism, cystic fibrosis, sickle cell disease, aminoacidopathies, organic acidurias, mitochondrial beta-oxidation disorders, and, lately, SCID). SCID was included in the Catalonian NBS program in January 2017, and data from the first 24 months following its implementation are presented here.

\section{MATERIALS AND METHODS \\ Population and Data Collection}

All consecutive DBS samples received as part of the universal NBS program in Catalonia between January 2017 and December 2018 were analyzed $(n=130,903)$. Samples with the following characteristics were excluded: collection time before $44 \mathrm{~h}$ or after 7 days of life, transfusions, poor DNA amplification, and poor quality or blood amount. Ultimately, this study was performed in 129,614 newborns.

Demographics (birth date, date of sample collection, parents' origin, newborn sex, gestational age, and birth weight) were electronically collected. Extremely preterm newborns were defined as those with a gestational age $<32$ weeks, preterm newborns $\geq 32$ and $<37$ weeks, and term newborns $\geq 37$ weeks. Low birth weight in term babies was defined as $<2,500 \mathrm{~g}$ and normal birth weight as $\geq 2,500 \mathrm{~g}$.

From 1 January to 30 June 2017, newborns $(n=33,040)$ underwent SCID screening as part of a 6-month prospective implementation pilot study that validated our approach. However, in January 2018, we decided to update the decision algorithm (Figure 1), lowering the retest cutoff from 34 to 24 copies/ $\mu \mathrm{L}$. The results from newborns screened in 2018 were then evaluated ( $n=64,092 ; 63,393$ after applying exclusion criteria).

The study was approved by the Government of Catalonia (Departament de Salut, Generalitat de Catalunya) for its NBS program. Specific informed consent was obtained from all 


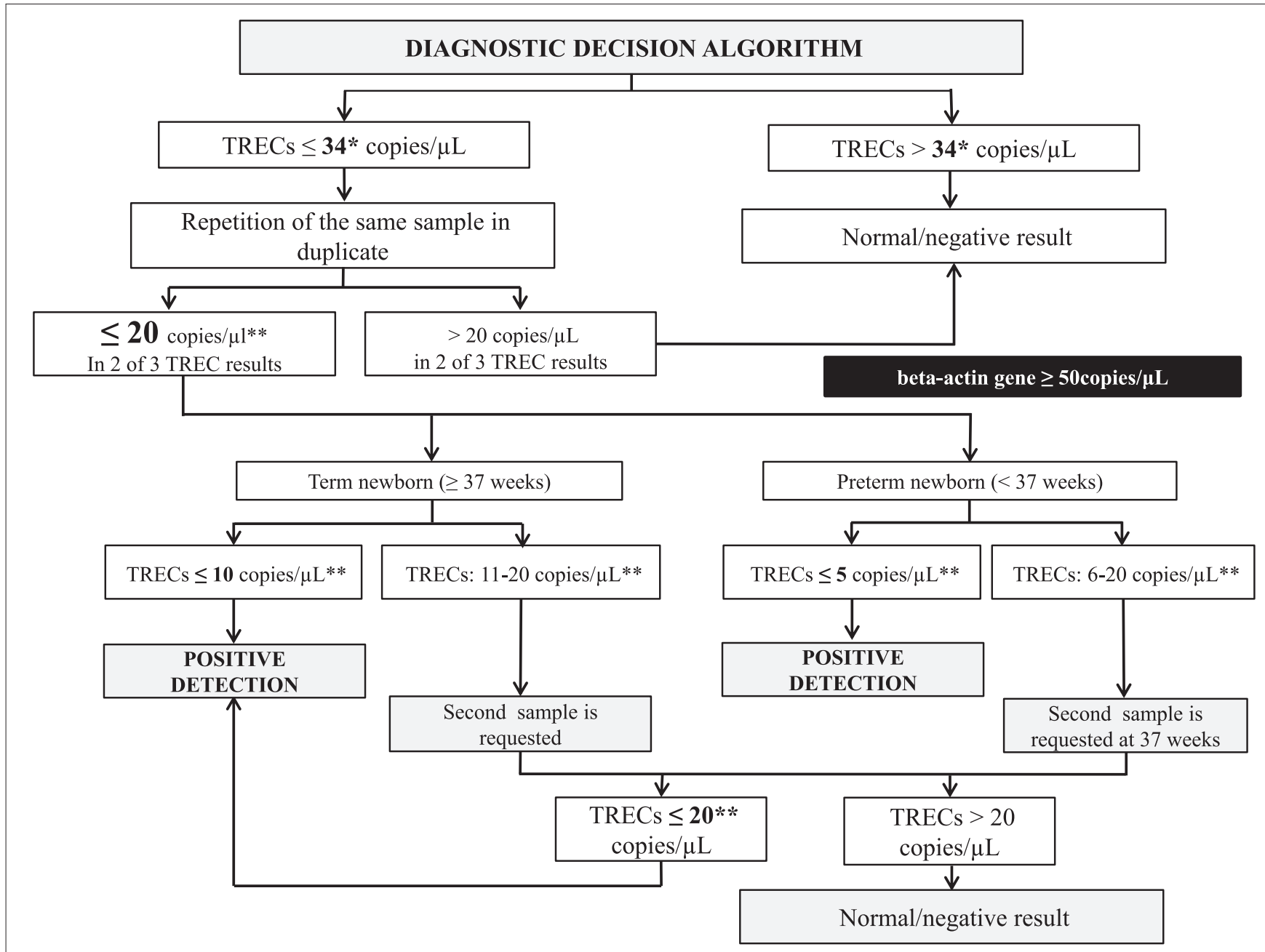

FIGURE 1 | SCID NBS detection decision algorit hm. *The retest cutoff was changed from 34 to 24 copies/ $\mu \mathrm{L}$ in 2018 . **If beta-actin gene <50 copies/ $\mu \mathrm{L}$ a second sample was requested because the sample was considered of unsatisfactory quality. TRECs, T-cell receptor excision circles.

individual participants included in the study whose genetic evaluation was needed.

\section{Sample Testing}

Quantification of TRECs in DBS (1.5-mm diameter spot) was performed according to the commercial Enlite Neonatal TREC kit instructions (PerkinElmer, Turku, Finland). The EnLite TREC kit is a combination of PCR-based nucleic acid amplification and time-resolved fluorescence resonance energy transfer (TR-FRET)-based detection. The assay detects two targets simultaneously: TRECs and beta-actin, which is used as the internal control to monitor specimen amplification in each test.

DBS punches were directly inserted in 96-well PCR plates using a Wallac DBS puncher (PerkinElmer). DNA was eluted in the first step, and the second step involved PCR amplification of TRECs and beta-actin as well as hybridization with target sequence-specific probes. The PCR plate was then read in a Victor EnLite fluorometer (PerkinElmer). A full calibration curve with blanks and three DBS calibrators was run in triplicate on each plate. A low-TREC control, a no-TREC control, a highTREC control, and a blank paper disk (with no blood content) were used as quality controls in each plate. Interpretation of the assay results uses fluorescence counts measured at 615, 665, and $780 \mathrm{~nm}$. Corrected fluorescence counts, TREC responses, and beta-actin responses for all reactions were calculated from the raw fluorescence counts. Calibrator responses were fitted against the ArcSinh transformed concentrations (copies $/ \mu \mathrm{L}$ ) using unweighted linear regression. The Enlite software generated a calibration curve and the sample and DBS control concentrations (copies/ $\mu \mathrm{L}$ ) in each run.

\section{Definition and Interpretation of Results}

Before implementing the protocol, the method was verified (repeatability, reproducibility, limit of detection, limit of quantification, sensitivity, and specificity) and these parameters were successfully compared with those stated in the kit insert (Supplementary Material S1). In addition, available NBS 


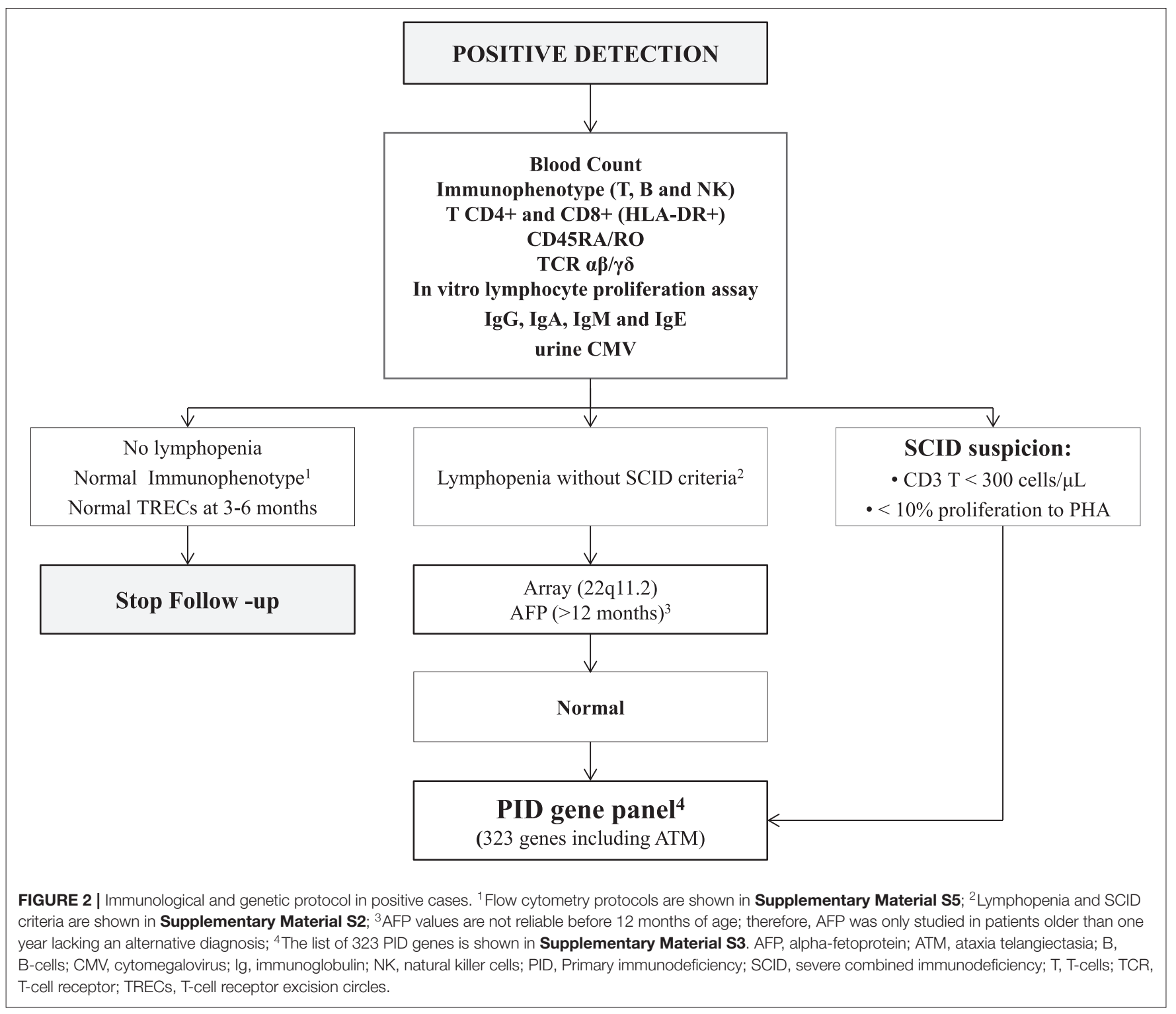

samples from children with a known SCID diagnosis in Catalonia in the last 5 years were analyzed as positive controls $(n=6$; median, range TREC copies/ $\mu \mathrm{L}: 2,2-4$ ), as well as five other positive samples from the SCID Newborn Screening Quality Assurance Program-Proficiency Testing Program provided by the CDC (Centers for Disease Control and Prevention, Atlanta, USA).

After reviewing the decision algorithms from other NBS programs with previous experience of this disease, we decided to start the pilot study with the algorithm used by Audrain et al. (6), which had a threshold of $>34$ copies/ $\mu \mathrm{L}$ (Figure 1 ).

Samples whose TREC value was $\leq 34$ copies/ $\mu \mathrm{L}$ were retested in duplicate. If two of the three values were $\leq 20$ copies $/ \mu \mathrm{L}$, a second sample was requested. Samples with TRECs $\leq 5$ copies $/ \mu \mathrm{L}$ (preterm infants) or $\leq 10$ copies/ $\mu \mathrm{L}$ (term newborns) in the first sample (both with beta-actin gene $\geq 50$ copies/ $\mu \mathrm{L}$ ), as well as analyses with TRECs $\leq 20$ copies/ $\mu \mathrm{L}$ in the second sample, were considered as positive detection (retest cutoff changed from 34 to 24 copies/ $\mu \mathrm{L}$ in 2018). These positive detections were notified to the SCID Clinical Reference Unit (SCID-CRU) to initiate clinical and immunological evaluation.

The retest after the first sample rate (retest rate), requested second sample rate, and SCID-positive detection rate (positive detections) were calculated. Based on these results, the algorithm was reevaluated. Validation of the results was carried out using Specimen Gate (Perkin Elmer) and Nadons (Limit4, Barcelona, Spain) software.

\section{Initial Clinical and Immunological Assessment at SCID-CRU}

Within the first 7 days after detection, all positive cases were referred to SCID-CRU, where clinical and immunological assessment was performed per protocol (Figure 2). Complete family and medical histories were recorded, and 
a meticulous physical examination was carried out. In addition, psychological support was offered to parents, starting with this first visit.

Several tests were performed: T, B, and NK cell immunophenotyping, expression of CD45RA/RO, TCR $\alpha \beta$, and $\gamma \delta$, HLA-DR+ expression on $\mathrm{T}$ lymphocytes, in vitro lymphocyte proliferation, and immunoglobulins including IgE. SCID criteria (Supplementary Material S2) were defined as reported by Kwan et al. (2). In cases of non-SCID lymphopenia, a consultation was scheduled with the geneticist for clinical evaluation and array-based comparative genomic hybridization (CGH-array) studies. If these results were normal, a genetic panel covering most PID was performed. We used a customdesigned next-generation sequencing (NGS)-based panel that targets 323 genes (Supplementary Material S3), including most of the known PID-causing genes according to the 2017 IUIS (International Union of Immunological Societies) classification, and other genes recently described as causing PID (20). This panel has been successfully used in our laboratory over the last few years for genetic diagnosis of PID (21).

\section{Statistical Analysis}

Statistical analyses were performed using SPSS software version 23.0 (SPSS Inc., Chicago, Illinois). Given the non-parametric distribution of the data, the Mann-Whitney $U$ or KruskalWallis test were used for group comparison analyses (25 and 75th percentiles in brackets). Two-tailed statistical analysis was performed, and differences were considered statistically significant at $p<0.05$.

\section{RESULTS}

\section{Demographics}

Of the 129,614 newborns screened after applying the exclusion criteria (initial $n=130,903$ ), $51.5 \%$ were male and $48.5 \%$ were female. The median age at sample collection was $50 \mathrm{~h}$ of life (interquartile range [IQR], 49-60). Median gestational age was 39 weeks (38-40): $0.6 \%$ were extremely preterm newborns $(n=$ $830), 5.9 \%$ preterm $(n=7,561)$, and $93.5 \%$ term newborns ( $n$ $=121,223)$. Median birth weight in term newborns was $3,300 \mathrm{~g}$ (3,010-3,590) (Table 1).

Median TREC value in the study population was 104 copies/ $\mu \mathrm{L}$ (IQR: $68-162$ ), with 20 copies/ $\mu \mathrm{L}$ being the 0.7 th percentile. Median TREC values showed statistically significant differences in relation to the patients' sex, gestational age, and birth weight (only assessed in term babies). Median TREC values were 98 (64-152) and 111 (73-173) in males and females, respectively $(p<0.05)$. In relation to gestational age, the median TREC value demonstrated a progressive increase: 70 (43127), 96 (62-155), and 105 (68-162) copies/ $\mu \mathrm{L}$ in extremely preterm, preterm, and term newborns, respectively $(p<0.05)$ (Supplementary Material S4). Regarding birth weight in term newborns, median TREC was 94 (61-145) and 105 (69-163) copies/ $\mu \mathrm{L}$ in low and normal birth weight babies, respectively $(p<.05)$ (Table 1$)$.
TABLE 1 | Demographic data and TREC values in the study population.

\begin{tabular}{|c|c|c|c|}
\hline Sample size & $n=129,614^{a}$ & $\begin{array}{c}\text { TRECs, } \\
\text { copies/ } \mu \mathrm{L}, \\
\text { median (IQR) }\end{array}$ & $p$-value ${ }^{c}$ \\
\hline All newborns & & $104(68-162)$ & - \\
\hline \multicolumn{4}{|l|}{ Sex } \\
\hline Male & $51.5 \%(66,751)$ & $98(64-152)$ & $<0.05$ \\
\hline Female & $48.5 \%(62,863)$ & $111(73-173)$ & \\
\hline $\begin{array}{l}\text { Sample collection, hours, } \\
\text { median (IQR) }\end{array}$ & $50(49-60)$ & - & - \\
\hline $\begin{array}{l}\text { Gestational age, weeks, } \\
\text { median (IQR) }\end{array}$ & $39(38-40)$ & & \\
\hline $\begin{array}{l}\text { Extremely preterm, <32 } \\
\text { weeks, \% (n) }\end{array}$ & $0.6 \%(830)$ & $70(43-127)$ & $<0.05$ \\
\hline $\begin{array}{l}\text { Preterm, 32-36 weeks, \% } \\
\text { (n) }\end{array}$ & $5.9 \%(7,561)$ & $96(62-155)$ & \\
\hline Term, $\geq 37$ weeks, \% (n) & $93.5 \%(121,223)$ & 105 (68-162) & \\
\hline $\begin{array}{l}\text { Birth weight in term } \\
\text { newborns }^{b} \text {, grams, } \\
\text { median (IQR): }\end{array}$ & $3300(3,010-3,590)$ & & \\
\hline Low birth weight, \% (n) & $3.4 \%(4,385)$ & $94(61-145)$ & $<0.05$ \\
\hline Normal weight,\% (n) & $96.6 \%(114,777)$ & 105 (69-163) & \\
\hline
\end{tabular}

a Sample size after applying exclusion criteria; ${ }^{b}$ Term newborns ( $\left.n=121,223\right) .{ }^{c}$ MannWhitney $U$ or Kruskal-Wallis test were used for group comparisons ( $p$-value calculated at a $5 \%$ significance level). IQR, interquartile range.

\section{Cutoff Values and Rates}

The overall results from 2017 to 2018 were retest rate $2.4 \%$, requested second sample rate $0.23 \%$, and positive detection rate $0.02 \%$ (Figure 3).

With the application of the initial TREC retest cutoff of 34 copies/ $\mu \mathrm{L}$ (3rd percentile in the 2017 population), results from the 66,214 samples analyzed during 2017 were as follows: retest rate $3.34 \%(n=2212)$, requested second sample rate $0.21 \%(n=$ $138)$, and positive detection rate $0.02 \%(n=16)$.

After reducing the retest cutoff to 24 copies/ $\mu \mathrm{L}$ (1st percentile), the retest rate was $1.4 \%(n=898)$. The detection cutoff of 20 copies/ $\mu \mathrm{L}$ remained the same ( 0.7 th percentile), and the requested second sample and positive detection rates were similar to those of 2017.

\section{Positive Detections}

There were 30 positive detections in the 129,614 screened newborns: $40 \%(n=12)$ were detected in the first sample and $60 \%(n=18)$ in the second, with $50 \%(n=15)$ of detections occurring in males. There was no history of maternal immunosuppression in any of the positive cases.

Newborns with positive detection were referred to the SCID-CRU per protocol. The various diagnoses in the 30 patients were as follows (Figure 4): SCID (1/30), partial 22q11 DiGeorge syndrome (5/30), idiopathic lymphopenia (3/30), chylothorax (2/30), prematurity (2/30), and Down syndrome $(1 / 30)$. Laboratory and clinical data of non-SCID TCL patients are described in Table 2. Nine patients were considered to have false-positive results (i.e., initially low TRECs and normal 


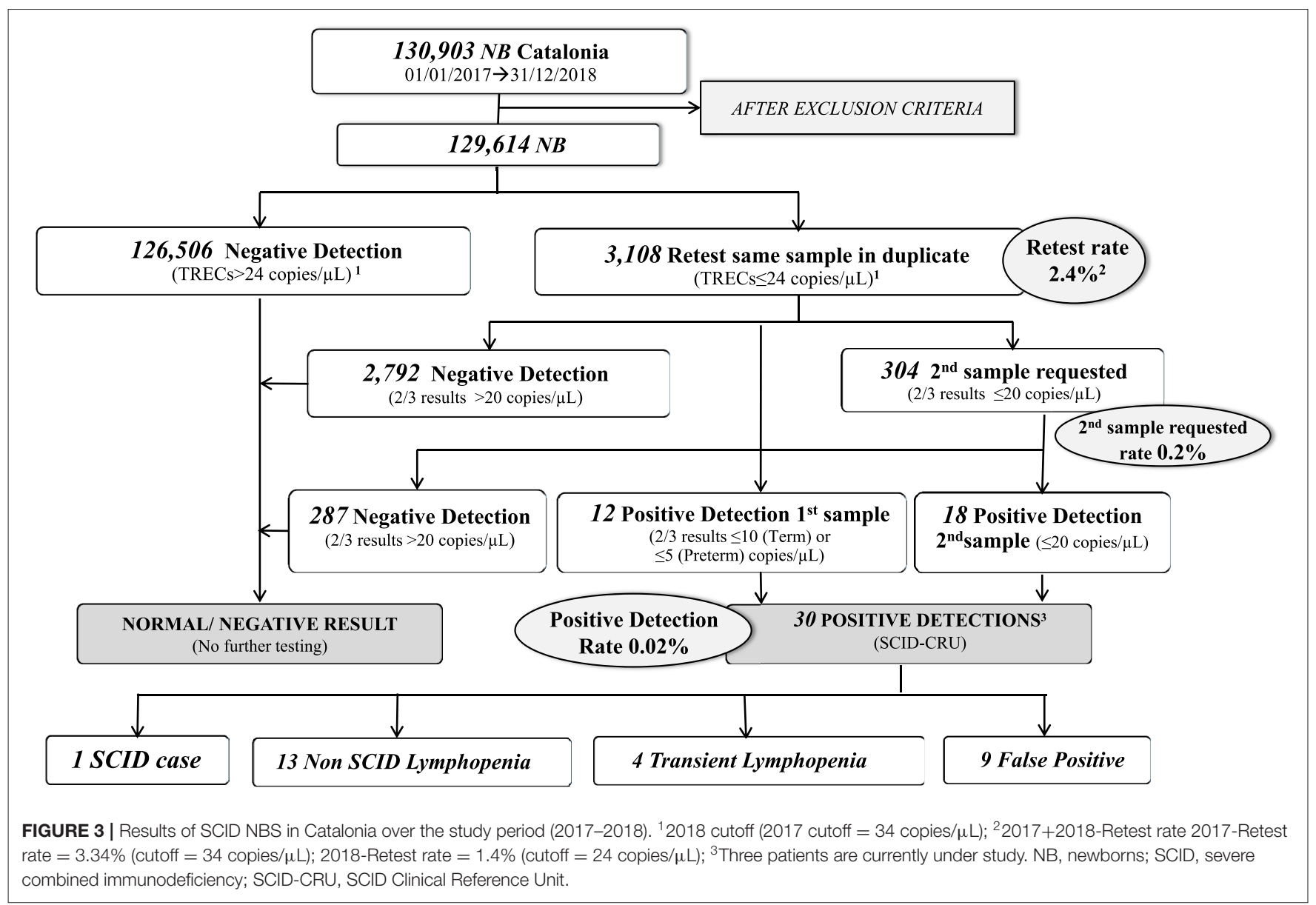

lymphocyte count, with normalization of TRECs between 3 and 6 months of life), four patients had transient lymphopenia (i.e., initially low TRECs and low lymphocyte count, with recovery in the following months), and three patients are currently under study (SCID excluded, but requiring additional TREC testing at 3 and 6 months of life). The incidence of clinically significant non-SCID TCL was 1 case in 10,069 newborns (43\% of positive detections).

Thus, the incidence of SCID was 1 in 130,903 newborns. The SCID patient was a Caucasian male of 35 weeks' gestation with $0 / 0$ TREC copies/ $\mu \mathrm{L}$ in DBS. Lymphocyte count was 0.4 $\times 10^{9} / \mathrm{L}$ with a T-B- NK+ phenotype and an absent in vitro proliferative response to mitogens. Whole exome sequencing was performed, but no causative mutations were found. However, a variant of uncertain significance was identified in the LIGlgene. The patient underwent HSCT at the age of 2 months of life using reduced intensity conditioning, with a good clinical outcome and immunological reconstitution.

\section{DISCUSSION}

This study reports the Catalonian NBS experience for SCID detection based on TREC quantification, and enables detection of non-SCID lymphopenic conditions in addition to SCID. After a 2-year experience and more than 130,000 newborns screened, one SCID patient and 13 other conditions were identified. In addition, we were able to consolidate our detection strategy and the characterization, diagnosis, and follow up protocol for positive cases.

As a pioneer initiative in Europe, we encourage the definition of a single technical approach for SCID newborn screening to facilitate future comparative studies between different countries. With this in mind, we decided to implement the commercially available EnLite Neonatal TREC kit (Perkin Elmer, Turku, Finland), which has been validated elsewhere and has shown comparable median TREC values between different studies $(4,6$, 22, 23). This kit allows for DNA elution and gene amplification in a single process, as well as standardization and reproducibility according to ISO 15189 standards. With the aim of minimizing the previously described inter-lot variability (6), we established a retest cutoff higher than the request second sample cutoff, which provided a range between the retest and detection cutoffs. The initial retest cutoff was 34 copies $/ \mu \mathrm{L}$ (3rd percentile), which was changed to 24 copies/ $\mu \mathrm{L}$ (1st percentile) in 2018, whereas the request second sample cutoff was maintained at 20 copies/ $\mu \mathrm{L}$.

The initial retest cutoff of 34 copies $/ \mu \mathrm{L}$ ( $3 \mathrm{rd}$ percentile) led to a retest rate of $3.34 \%$, which was considered too high in comparison with data from other authors (24). Hence, we 
then evaluated the first percentile from our population (24 copies/ $\mu \mathrm{L}$ ) as a new retest cutoff. We found that 1,477 of 1,478 babies with initial TREC values between 34 and 25

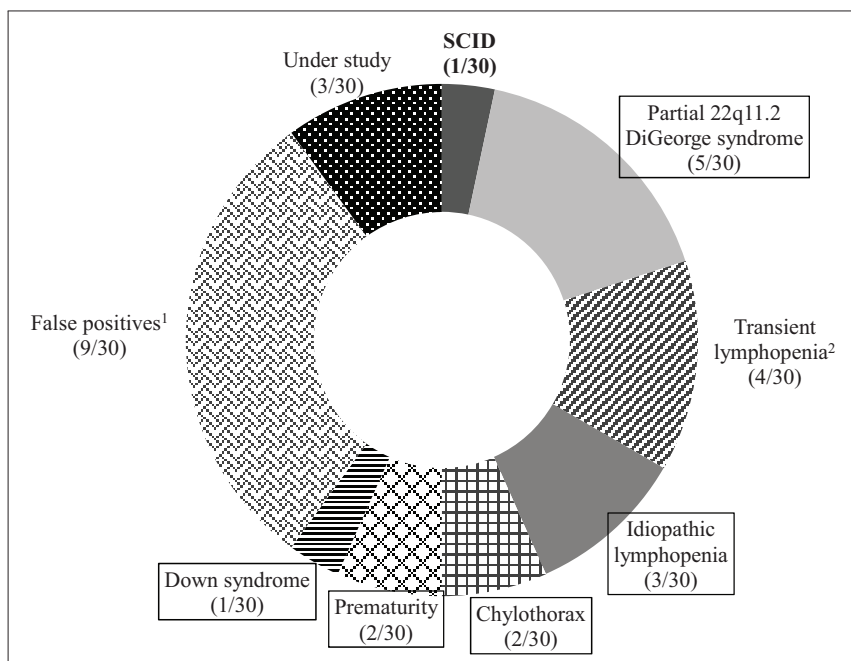

FIGURE 4 | Final diagnoses in patients testing positive in Catalonia (2017-2018). ${ }^{1}$ False-positives due to initially normal lymphocyte count with normalization of TRECs between 3 and 6 months of life; ${ }^{2}$ Transient lymphopenia due to initially low lymphocyte count with recovery in the following months. Final diagnosis of the 13 non-SCID lymphopenic patients are now boxed. SCID, severe combined immunodeficiency. copies/ $\mu \mathrm{L}$ were normal: 1,455 (98.4\%) after duplicate repetition and $22(1.6 \%)$ after a second DBS analysis. Only one baby within this range was referred for study in the SCID-CRU and the diagnosis was not a case of SCID, the objective of this screening. Following this analysis, we decided that it was reasonable to switch to 24 copies/ $\mu \mathrm{L}$ as the TREC repeat cutoff, implemented since 2018. That change reduced the repetition rate to $1.4 \%$, now comparable to the rate described by others (24) and led to a reduction in the screening cost per patient.

Our internal algorithm showed a second sample request rate and positive detection rate of 0.2 and $0.02 \%$, respectively, in keeping with the rates in previous studies, as summarized in the review by van der Spek et al. (24). In addition, the percentiles of our population corresponding to each cutoff are comparable to those of other diseases included in our program, such as congenital hypothyroidism, phenylketonuria, and other metabolic diseases. Of note, the method was unable to adequately quantify beta-actin copies in some of the external quality control samples from the CDC program, a shortcoming that may be related to PCR inhibition, as was suggested by the CDC itself. Nonetheless, this limitation did not affect the results of SCID screening.

Several studies have used combined TREC and KREC screening assays to simultaneously detect T- and B-cell defects $(18,25)$, including a pioneer study performed in Spain by de Felipe et al. (26), but we decided to focus on classical SCID patients who completely fulfill the NBS criteria, in accordance with the experience in the United States $(2,5)$. Although

TABLE 2 | Clinical and laboratory data in non-SCID TCL patients.

\begin{tabular}{|c|c|c|c|c|c|c|c|c|c|}
\hline Patient & $\begin{array}{l}\text { Gestational } \\
\text { age }\end{array}$ & Sex & $\begin{array}{l}\text { Prenatal } \\
\text { diagnosis }\end{array}$ & $\begin{array}{l}\text { TREC levels } \\
\text { (copies } / \mu \mathrm{L} \text { ) }\end{array}$ & $\begin{array}{l}\text { Lymphocyte } \\
\text { count, (x109/L)* }\end{array}$ & $\begin{array}{l}\text { Lymphocyte } \\
\text { subsets:T cells/B } \\
\text { cells/NK cells } \\
\left(\times 10^{9} / L\right)^{* *}\end{array}$ & $\begin{array}{l}\text { Lympho- } \\
\text { proliferationassay }\end{array}$ & $\begin{array}{l}\text { Clinical features at } \\
\text { birth }\end{array}$ & Diagnosis \\
\hline 1 & 40 & Female & No & 17 & 4.2 & 2.1/19.9/1.1 & Normal & None & $22 \mathrm{q} 11.2 \mathrm{DS}$ \\
\hline 2 & 40 & Female & No & 5 & 5.4 & $1.6 / 1.1 / 2.3$ & Normal & None & $22 q 11.2$ DS \\
\hline 3 & 34 & Female & Yes & 5 & 2.6 & $1.1 / 0.6 / 0.7$ & Normal & Congenital heart disease & $22 \mathrm{q} 11.2 \mathrm{DS}$ \\
\hline 5 & 41 & Female & No & 17 & 1.7 & $1.2 / 0.04 / 0.3$ & Normal & None & $\begin{array}{l}\text { 22q11.2 } \\
\text { duplication }\end{array}$ \\
\hline 6 & 40 & Male & No & 14 & 3.7 & 2.2/0.6/0.6 & Normal & None & $\begin{array}{l}\text { Idiopathic } \\
\text { Iymphopenia }\end{array}$ \\
\hline 9 & 34 & Female & Yes & 6 & 7.8 & NA & Normal & Congenital Chylothorax & Chylothorax \\
\hline 10 & 34 & Female & Yes & 4 & 7.4 & NA & Normal & Hydrops fetalis & Chylothorax \\
\hline 11 & 32 & Male & No & 7 & 2.7 & 2.9/0.8/0.2 & Normal & None & Prematurity \\
\hline 12 & 29 & Male & No & 18 & 4.4 & 2.9/1/0.2 & Normal & None & Prematurity \\
\hline 13 & 31 & Female & No & 15 & 3 & NA & Normal & Esophageal atresia & $\begin{array}{l}\text { Down } \\
\text { syndrome }\end{array}$ \\
\hline
\end{tabular}

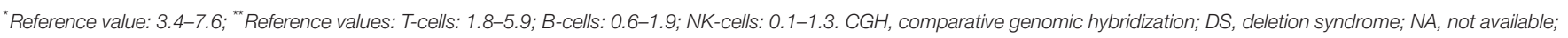
NBS, newborn screening; SCID, severe combined immunodeficiency; TREC, T-cell receptor excision circle. 
inclusion of KREC values to the TREC assay would enable the detection of patients with hypomorphic mutations leading to a leaky SCID phenotype and delayed-onset adenosine deaminase (ADA) deficiency $(14,25,27)$, it might also result in an increase in false-positive testing and higher recall and retest rates.

Our data support the previously reported influence of gestational age and sex on TREC values (27-30). Thus, in the case of low values in preterm babies or low-birth-weight term newborns (i.e., TREC values above 5 copies/ $\mu \mathrm{L}$ in preterm babies and 10 copies/ $\mu \mathrm{L}$ in term newborns), cautious evaluation and a request for a second sample are needed at term and when birth weight reaches $>2,500 \mathrm{~g}$, respectively. To our knowledge, there is no explanation for the significantly higher TREC values seen in female newborns; however, these differences have been described previously in a cohort study reported by Rechavi et al. (4) Further studies may provide data to define the reason for these differences and their clinical implications, if any.

With regard to gestational age, median TREC levels in our cohort rose significantly from 28 to 32 weeks gestation in accordance with T-cell maturation in this period, a wider period of time than those reported by other authors $(4,18,26)$ (Supplementary Material S4).

Overall, there were 30 positive detections in the 129,614 newborns screened (130,903 before applying the exclusion criteria). All were referred to the SCID-CRU per protocol. Nine patients were considered to have false-positive results due to initially normal total lymphocyte and CD3+ counts with normalization of TRECs between 3 and 6 months of life, whereas four others had transient lymphopenia at the beginning with recovery in the following months, and three are currently under study [none of the three patients under study met the SCID criteria, as defined by Kwan et al. (2)].

The diagnosis of the T-B-NK+ SCID patient permitted timely referral to a specialized treatment center for HSCT, after which $\mathrm{T}$ and $\mathrm{B}$ lymphocyte counts normalized with fully reconstituted function, eliminating the need for IgG supplementation at 1 year of age.

The incidence of SCID found in this study (1 in 130,903) is lower than the reported rates from other areas $(2,4,24)$. However, we anticipate that a more robust incidence will be established when the population analyzed reaches 200,000 newborns. In this line, a retrospective study in our region demonstrated an incidence of classically diagnosed SCID of 1 in 57,000 newborns (unpublished data). In addition to the SCID patient, 13 infants with non-SCID TCL were identified, yielding an incidence of 1 in 10,069 newborns (43\% positive detections). This is similar to the rate reported by others (31). The diagnostic distribution was also comparable. It is worth mentioning that early detection of patients with 22q11.2 deletion syndrome allowed a prompt intervention to anticipate complications, as reported by our group (Mol Genet Genomic Med, accepted).

Of note, one of the strengths of our SCID NBS approach is the psychological support offered to the families. A positive result in any NBS program is a cause of concern for parents at this vulnerable time because of an unfamiliarity with these illnesses, the young age of the patients, and the stress on families after a birth. Therefore, in neonates initially testing positive, a psychologist provided support starting from the first contact with the family in a joint visit with the pediatric immunologist. The psychologist's task was to guarantee that parents were able to understand and assimilate the information about the possible diagnosis and the process to follow in the event that it was confirmed. The family was offered a space for containment and support for the impact of the diagnosis, and strategies were activated to control anxiety. The psychologist maintained contact to ensure adherence to the treatments prescribed and compliance with the control visits and tests. Support was intensified in the most complicated moments, such as hospital admission and isolation, and parents were accompanied in all phases related to transplantation. Several cases of parental anxiety were detected and appropriately managed. In the yearly evaluation, families rated the psychological support in our NBS program very favorably.

The main limitation of this study is the relatively small number of newborns screened, although the similarity between our results and those reported in larger cohorts supports their validity. In addition, retrospective data show an incidence of SCID in our region similar to that of other Western European countries $(6,18)$ and the United States $(5)$.

To conclude, TREC quantification in DBS for SCID detection has been satisfactorily implemented in the Catalonian NBS program. Retesting, requested second samples, and positive detection rates were optimal with the current algorithm and similar to published data. Our results provide further evidence to support the inclusion of SCID in NBS programs in other regions and countries. Longer follow-up is needed to define the exact incidence of SCID in Catalonia.

\section{DATA AVAILABILITY STATEMENT}

The datasets generated for this study are available on request to the corresponding author.

\section{ETHICS STATEMENT}

The studies involving human participants were reviewed and approved by Comité Ético from Agència de Salut Pública (Catalonia). Written informed consent to participate in this study was provided by the participants' legal guardian/next of kin.

\section{AUTHOR CONTRIBUTIONS}

AA-R, AM-N, and PS-P designed the study. YQ and TC collected the data and participated in the analytical tools. AA-R and JM-S organized the database. AA-R and RL-G performed the statistical analysis. Results were analyzed and interpreted by AA-R, AR, AM-N, and PS-P. AA-R, AM-N, and PS-P wrote the first draft of the manuscript. MG-P, MM-G, JR, RC, SP-G, JG, JG-V, and JM-S wrote sections of the manuscript. All authors contributed to manuscript revision and read and approved the submitted version. 


\section{ACKNOWLEDGMENTS}

Dr. Marie Audrain (CHU de Nantes, Nantes, France) for contributing to the development of the algorithm. Mr. Antoni Comin, former Catalan Minister of Health, for his personal implication in the implementation of SCID NBS in Catalonia. Dr. Rosa Fernández, former Mother and Child Health Division Chief (Health Department) for her support and professionalism with the NBS Program of Catalonia. Mrs. Anna Fabregas, for providing psychological support to all patients's parents with positive results in the SCID NBS screening. Mrs. Marta Parellada, for her valuable administrative work. The Catalan Association for Primary Immune Defects, the BCN-PID Foundation, and

\section{REFERENCES}

1. Guthrie, R. Blood screening for phenylketonuria. JAMA. (1961) 178:863. doi: 10.1001/jama.1961.03040470079019

2. Kwan A, Abraham RS, Currier R, Brower A, Andruszewski K, Abbott $\mathrm{JK}$, et al. Newborn screening for severe combined immunodeficiency in 11 screening programs in the United States. JAMA. (2014) 312:72938. doi: 10.1001/jama.2014.9132

3. Chien YH, Chiang SC, Chang KL, Yu HH, Lee WI, Tsai LP, et al. Incidence of severe combined immunodeficiency through newborn screening in a Chinese population. J Formos Med Assoc. (2015) 114:12-6. doi: 10.1016/j.jfma.2012.10.020

4. Rechavi E, Lev A, Simon AJ, Stauber T, Daas S, Saraf-Levy T, et al. First Year of Israeli Newborn Screening for Severe Combined Immunodeficiency - Clinical Achievements and Insights. Front. Immunol. (2017) 8:1448. doi: 10.3389/fimmu.2017.01448

5. Puck JM. Newborn screening for severe combined immunodeficiency and T-cell lymphopenia. Immunol Rev. (2019) 287:241-52. doi: 10.1111/imr.12729

6. Audrain MAP, Léger AJC, Hémont CAF, Mirallié SM, Cheillan D, Rimbert MGM, et al. Newborn screening for severe combined immunodeficiency: analytic and clinical performance of the $\mathrm{T}$ cell receptor excision circle assay in france (DEPISTREC Study). J Clin Immunol. (2018) 38:77886. doi: 10.1007/s10875-018-0550-7

7. Brown L, Xu-Bayford J, Allwood Z, Slatter M, Cant A, Davies EG, et al. Neonatal diagnosis of severe combined immunodeficiency leads to significantly improved survival outcome: the case for newborn screening. Blood. (2011) 117:3243-6. doi: 10.1182/blood-2010-08-300384

8. Buckley RH, Schiff SE, Schiff RI, Markert L, Williams LW, Roberts JL, et al. Hematopoietic stem-cell transplantation for the treatment of severe combined immunodeficiency. N Engl J Med. (1999) 340:50816. doi: 10.1056/NEJM199902183400703

9. Chan K, Puck JM. Development of a population-based screening for severe combined immunodeficiency. J Allergy Clin Immunol. (2005) 115:3918. doi: 10.1016/j.jaci.2004.10.012

10. Thomas C, Durand-Zaleski I, Frenkiel J, Mirallié S, Léger A, Cheillan D, et al. Clinical and economic aspects of newborn screening for severe combined immunodeficiency: DEPISTREC study results. Clin Immunol. (2019) 202:339. doi: 10.1016/j.clim.2019.03.012

11. King J, Hammarström L. Newborn screening for primary immunodeficiency diseases: history, current and future practice. J Clin Immunol. (2018) 38:5666. doi: 10.1007/s10875-017-0455-x

12. Routes J, Verbsky J. Newborn screening for severe combined immunodeficiency. curr allergy Asthma Rep. (2018) 18:34. doi: 10.1007/s11882-018-0783-9

13. Speckmann C, Neumann C, Borte S, la Marca G, Sass JO, Wiech E, et al. Delayed-onset adenosine deaminase deficiency: strategies for an early diagnosis. J Allergy Clin Immunol. (2012) 130:991-4. doi: 10.1016/j.jaci.2012.04.004 the PID group from the Catalan Societies of Pediatrics and Immunology for their invaluable help to implement SCID NBS in Catalonia. We are grateful to the laboratory technicians from the Newborn Screening Laboratory of Hospital Clínic de Barcelona and from the Immunology Division of Hospital Universitari Vall d'Hebron.

\section{SUPPLEMENTARY MATERIAL}

The Supplementary Material for this article can be found online at: https://www.frontiersin.org/articles/10.3389/fimmu. 2019.02406/full\#supplementary-material

14. Borte S, Wang N, Oskarsdóttir S, von Döbeln U, Hammarström L. Newborn screening for primary immunodeficiencies: beyond SCID and XLA. Ann N Y Acad Sci. (2011) 1246:118-30. doi: 10.1111/j.1749-6632.2011.06350.x

15. Puck JM. Laboratory technology for population-based screening for severe combined immunodeficiency in neonates: the winner is T-cell receptor excision circles. J Allergy Clin Immunol. (2012) 129:607-16. doi: 10.1016/j.jaci.2012.01.032

16. Modell V, Knaus M, Modell F. An analysis and decision tool to measure cost benefit of newborn screening for severe combined immunodeficiency (SCID) and related T-cell lymphopenia. Immunol Res. (2014) 60:14552. doi: 10.1007/s12026-014-8485-4

17. Ding Y, Thompson JD, Kobrynski L, Ojodu J, Zarbalian G, Grosse SD. Cost-effectiveness/cost-benefit analysis of newborn screening for severe combined immune deficiency in Washington State. J Pediatr. (2016) 172:12735. doi: 10.1016/j.jpeds.2016.01.029

18. Barbaro M, Ohlsson A, Borte S, Jonsson S, Zetterström RH, King J, et al. Newborn screening for severe primary immunodeficiency diseases in Sweden - a 2-years pilot TREC and KREC screening study. J Clin Immunol. (2017) 37:51-60. doi: 10.1007/s10875-016-0347-5

19. Van der Ploeg CPB, Blom M, Bredius RGM, van der Burg M, Schielen PCJI, Verkerk PH, et al. Cost-effectiveness of newborn screening for severe combined immunodeficiency. Eur J Pediatr. (2019) 178:721. doi: 10.1007/s00431-019-03346-3

20. Picard C, Bobby Gaspar H, Al-Herz W, Bousfiha A, Casanova JL, Chatila $\mathrm{T}$, et al. International Union of Immunological Societies: 2017 primary immunodeficiency diseases committee report on inborn errors of immunity. $J$ Clin Immunol. (2018) 38:96-128. doi: 10.1007/s10875-017-0464-9

21. Dieli-Crimi R, Martínez-Gallo M, Franco-Jarava C, Antolin M, Blasco L, Paramonov I, et al. Th1-skewed profile and excessive production of proinflammatory cytokines in a NFKB1-deficient patient with CVID and severe gastrointestinal manifestations. Clin Immunol. (2018) 195:4958. doi: 10.1016/j.clim.2018.07.015

22. Blom M, Pico-Knijnenburg I, Sijne-van Veen M, Boelen A, Bredius RGM, van der Burg $M$, et al. An evaluation of the TREC assay with regard to the integration of SCID screening into the Dutch newborn screening program. Clin Immunol. (2017) 180:106-10. doi: 10.1016/j.clim.2017.05.007

23. Adams SP, Rashid S, Premachandra T, Harvey K, Ifederu A, Wilson MC, et al. Screening of neonatal UK dried blood spots using a duplex TREC screening assay. J Clin Immunol. (2014) 34:323-30. doi: 10.1007/s10875-0140007-6

24. van der Spek J, Groenwold RH, van der Burg M, van Montfrans JM. TREC based newborn screening for severe combined immunodeficiency disease: a systematic review. J Clin Immunol. (2015) 35:416-30. doi: 10.1007/s10875-015-0152-6

25. Nourizadeh M, Shakerian L, Borte S, Fazlollahi M, Badalzadeh M, Houshmand $\mathrm{M}$, et al. Newborn screening using TREC/KREC assay for severe $\mathrm{T}$ and B cell lymphopenia in Iran. Scand J Immunol. (2018) 88:e12699. doi: 10.1111/sji.12699 
26. de Felipe B, Olbrich P, Lucenas JM, Delgado-Pecellin C, Pavon-Delgado A, Marquez J, et al. Prospective neonatal screening for severe $\mathrm{T}$ and $\mathrm{B}-$ lymphocyte deficiencies in Seville. Pediatr Allergy Immunol. (2016) 27:707. doi: 10.1111/pai.12501

27. Borte S, Puck J, Lorey F, McGhee SA, Hershfield MS, Fasth A, et al. KRECs but not TRECs identify patients with delayed-onset adenosine deaminase (ADA) deficiency in neonatal screening. J Clin Immunol. (2013) 33:673.

28. Routes JM, Grossman WJ, Verbsky J, Laessig RH, Hoffman GL, Brokopp $\mathrm{CD}$, et al. Statewide newborn screening for severe T-cell lymphopenia. JAMA. (2009) 302:2465-70. doi: 10.1001/jama.2009.1806

29. Vogel BH, Bonagura V, Weinberg GA, Ballow M, Isabelle J, DiAntonio L, et al. Newborn screening for SCID in New York state: experience from the first two years. J Clin Immunol. (2014) 34:289-303. doi: 10.1007/s10875-0140006-7

30. Verbsky JW, Baker MW, Grossman WJ, Hintermeyer M, Dasu T, Bonacci $B$, et al. Newborn screening for severe combined immunodeficiency; the Wisconsin experience (2008-2011). J Clin Immunol. (2012) 32:828. doi: 10.1007/s10875-011-9609-4
31. Amatuni GS, Currier RJ, Church JA, Bishop T, Grimbacher E, Nguyen AA, et al. Newborn Screening for Severe Combined Immunodeficiency and T-cell Lymphopenia in California, 2010-2017. Pediatrics. (2019) 143:e20182300. doi: 10.1542/peds.2018-2300

Conflict of Interest: The authors declare that the research was conducted in the absence of any commercial or financial relationships that could be construed as a potential conflict of interest.

Copyright (c) 2019 Argudo-Ramírez, Martín-Nalda, Marín-Soria, López-Galera, Pajares-García, González de Aledo-Castillo, Martínez-Gallo, García-Prat, Colobran, Riviere, Quintero, Collado, García-Villoria, Ribes and Soler-Palacín. This is an openaccess article distributed under the terms of the Creative Commons Attribution License (CC BY). The use, distribution or reproduction in other forums is permitted, provided the original author(s) and the copyright owner(s) are credited and that the original publication in this journal is cited, in accordance with accepted academic practice. No use, distribution or reproduction is permitted which does not comply with these terms. 